\title{
Disorders of lung matrix remodeling
}

\author{
Harold A. Chapman \\ Department of Medicine and Cardiovascular Research Institute, University of California at San Francisco, \\ San Francisco, California, USA
}

A set of lung diseases share the tendency for the development of progressive fibrosis ultimately leading to respiratory failure. This review examines the common pathogenetic features of these disorders in light of recent observations in both humans and animal models of disease, which reveal important pathways of lung matrix remodeling.

J. Clin. Invest. 113:148-157 (2004). doi:10.1172/JCI200420729.

Pulmonary fibrosis is a common consequence and often a central feature of many lung diseases. In some disorders fibrosis develops focally and to a limited degree. For example, in asthma and chronic obstructive pulmonary disease fibrotic changes occur around conducting airways where scarring may be important to the pathophysiology $(1,2)$. But fibrosis is not the dominant pathological feature of either disease. In contrast, a subset of lung diseases are particularly vexing because the degree of fibroproliferation and fibrosis is a dominant determinant of clinical outcome and yet for the most part current therapies are ineffective or only marginally effective. These diseases share in common the propensity for progressive fibrosis leading to respiratory failure and thus they are, in a sense, disorders of lung matrix remodeling. They also share common elements of pathobiology favoring matrix and architectural remodeling and disease progression,

The Science in Medicine series is supported in part by a generous grant from the Doris Duke Charitable Foundation.

Address correspondence to: Harold A. Chapman, Pulmonary and Critical Care Division, University of California at San Francisco, 513 Parnassus Avenue, San Francisco, California 94143-0130, USA. Phone: (415) 514-1210; Fax: (415) 502-4995; E-mail: halchap@itsa.ucsf.edu.

Conflict of interest: The author has declared that no conflict of interest exists.

Nonstandard abbreviations used: idiopathic pulmonary fibrosis (IPF); adult respiratory distress syndrome (ARDS); acute interstitial pneumonitis (AIP); usual interstitial pneumonitis (UIP); collagen vascular disease (CVD); bronchiolitis obliterans (BO); cryptogenic organizing pneumonia (COP); bronchoalveolar lavage (BAL); alveolar macrophage (AM); activated protein $\mathrm{C}$ (APC); sonic hedgehog (SHH); epidermal growth factor (EGF); bone morphogenic protein (BMP); epithelial-mesenchymal transition (EMT); latency-associated peptide (LAP); thrombospondin-1 (TSP-1); latent TGF-binding protein (LTBP); plasminogen activator inhibitor-1 (PAI-1); protease-activated receptor-1 (PAR-1); connective tissue growth factor (CTGF); monocyte chemotactic protein-1 (MCP-1); surfactant protein C (SPC); single nucleotide polymorphism (SNP); nonspecific interstitial pneumonia (NSIP). especially repeated epithelial cell death, and for all of these reasons are grouped together (Table 1 ). The disorders listed in Table 1 are mostly diseases of the modern era. This grouping underrepresents causes of severe fibrotic lung disease in regions of heavy dust exposure and endemic tuberculosis. But better control of infections, work conditions, longer life spans, and new medical technology such as mechanical ventilation and organ transplantation have resulted in disorders of lung matrix remodeling becoming more prevalent. A patient over the age of 50 presenting with several months of dyspnea without signs of infection, bilateral infiltrates on chest X-ray, and a restrictive pattern of pulmonary function abnormalities now has almost a $40 \%$ chance of having idiopathic pulmonary fibrosis (IPF) as the underlying disease (3).

Differences in the tempo and sites of disease progression among these disorders (Table 1) tend to obscure common clinical features. The adult respiratory distress syndrome (ARDS) is a dramatic example of acute, diffuse alveolar damage and non-cardiogenic pulmonary edema. But an important issue for longterm survival is the degree of parenchymal fibrosis and loss of lung function (4-6). This development during the acute or subacute setting, heralded by a progressive increase in pulmonary vascular resistance, leads to the hallmark clinical features of pulmonary fibrosis common to all of these disorders: persistent patchy radiographic infiltrates, reduced lung compliance indicative of restrictive lung disease, progressively impaired gas exchange, and ultimately cor pulmonale. The same applies to acute interstitial pneumonitis (AIP), which has a similar time course and pathology as ARDS but no known precipitating event (7). In stark contrast to the dramatic nature of ARDS and AIP is the prototypic fibrotic lung disease, IPF, a diagnosis more recently restricted to the histological pattern, usual interstitial pneumonitis (UIP) (8). In this disease, there is virtually no evidence of an acute process on plain chest radi- 


\section{Table 1}

Disorders of lung matrix remodeling

Idiopathic pulmonary fibrosis

Adult respiratory distress syndrome

Fibrosis with collagen vascular disease

Cryptogenic organizing pneumonia

Bronchiolitis obliterans, transplant associated

Sarcoidosis

Histiocytosis X (eosinophilic granuloma)

Hermansky-Pudlak syndrome

Disorders where fibrosis is frequently prominent but not usually a cause of death: asbestosis, hypersensitivity pneumonitis, drug-induced lung disease, localized fibrosis around airways in asthma and emphysema, and lung irradiation.

ographs. Fibrosis associated with well-defined collagen vascular diseases (CVDs) such as scleroderma fall somewhere between ARDS and IPF, with elements of acute and chronic inflammation and injury. Three of these disorders are strikingly bronchocentric: sarcoidosis, eosinophilic granuloma, and bronchiolitis obliterans (BO). Accordingly, their clinical features are a mixture of both restriction (from progressive fibrosis) and obstruction (from airway narrowing). Although respiratory failure is very uncommon in sarcoidosis and variable in eosinophilic granuloma, both disorders are found as indications for lung transplantation in the setting of end-stage fibrosis (9). Progressive intralumenal and airway matrix organization in $\mathrm{BO}$ is a major cause of disability and fatality following lung or bone marrow transplantation (10).

In spite of their clinical distinctions, the disorders of matrix remodeling share a common paradigm of disease progression: provisional matrices formed in the context of injury emit signals to activate an inflammatory response and epithelial cells, provoking ingrowth and/or expansion of connective tissue elements that lead to persistent and at times permanent matrix reordering. This is highlighted in Figure 1, which illustrates the loose matrices of lesional activity in UIP, BO, and cryptogenic organizing pneumonia (COP) being covered by epithelial cells and invaded by inflammatory cells and fibroblasts. Several comprehensive reviews focused on the distinguishing clinical, radiographic, and histological patterns among these diseases have been recently published $(3,8,11)$. This review will focus on our current understanding of the pathobiology common to the progressively fibrotic lung diseases (Table 1 ). Understanding molecular mechanisms driving the fibrotic process for even one of these disorders may empower new efforts to monitor and treat the group of disorders where lung matrix remodeling dominates.

\section{Historical perspectives and emergence of current concepts}

Role of inflammation. A seminal set of findings in the mid-1970s and early 1980s, based on the introduction into clinical research of bronchoalveolar lavage (BAL), revealed that patients with UIP or any of the related set of chronic fibrotic disorders (Table 1) have a persistent alveolitis $(12,13)$. This was found to be true whether or not any evidence of edema or inflammation was detectable on the plain radiograph, leading to the paradigm that early and persistent inflammation was the cause of injury and subsequent development of fibrosis. This idea was seemingly corroborated by thenemerging high resolution chest imaging techniques: changes consistent with edema and inflammation, i.e., hazy increased densities on the radiograph not obscuring the underlying architecture (termed "ground glass" changes), were found to be very common, even in UIP, and the extent of these changes tended to correlate with less fibrotic, early phases of disease (8). Because the alveolar space could be readily sampled in these patients, this paradigm also spawned many studies examining the levels of inflammatory mediators, biomarkers of injury, and cytokines thought to be relevant to matrix remodeling and fibrosis. Over time, several paradigms have emerged. One paradigm is that chemokines, cytokines, and other mediators found to be upregulated in the BAL of patients with fibrotic processes act at more than one point in the inflammatory response. Leukotrienes promote the inflammatory response and impair barrier function of the lung epithelium, perhaps important to host defense (14). Cysteinyl-leukotrienes such as LTC4 also stimulate fibroblast proliferation and collagen production, a potential mechanism for an exaggerated healing response (15). Other chemokines exhibit similar profiles (16). Conversely, a cytokine strongly linked to fibroblast activation and matrix production, TGF- $\beta_{1}$, also regulates lung permeability in response to acute injury (17). A second paradigm is that there are pat-

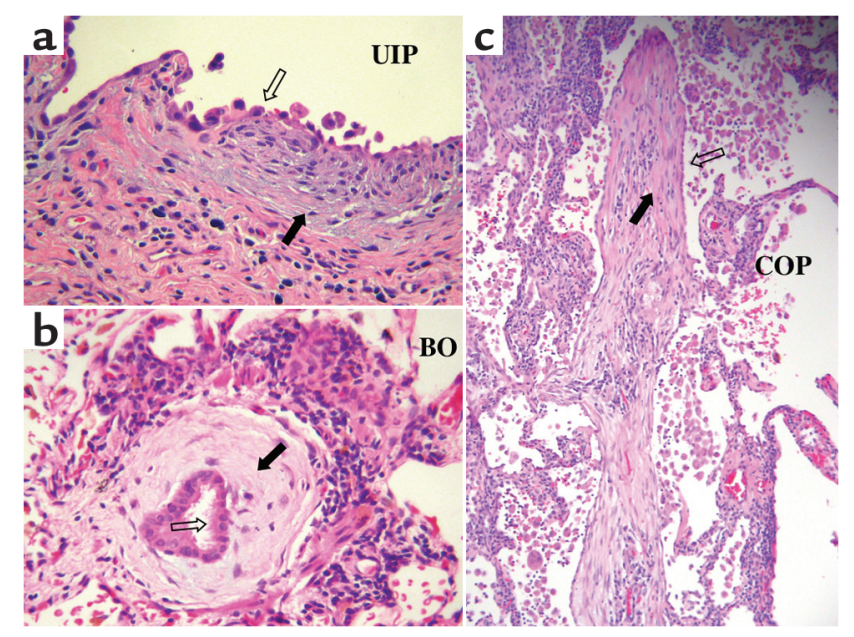

Figure 1

Photomicrographs of active matrix remodeling in: (a) UIP, (b) BO, and (c) COP. Filled arrows point to areas of active matrix remodeling in each disorder. Open arrows point to airway or alveolar epithelial cells overlying the remodeling matrix. Images were kindly provided by Kirk Jones, Department of Pathology, University of California, San Francisco, San Francisco, California, USA. Magnifications: (a, b) $\times 400$; (c) $\times 100$. 
Table 2

Profibrotic mediators of human lung fibrosis

Chemokines
IL-1 $\beta$
CXC ligand epithelial neutrophil activating protein-78
MCP-1A
Leukotrienes
Leukotrienes C4, E4A
Cytokines
TGF- $\alpha$, TGF- $\beta^{A}$
IGF-1
TNF $\alpha$
Connective tissue growth factor
PDGF
Endothelin-1 ${ }^{\text {A }}$
Thrombin ${ }^{A}$
PAl- $1^{\text {A }}$
APC inhibitor
Tissue inhibitor of metalloprotease- 1

BAL fluids may also show relatively low levels of PGE2, IFN- $\gamma$, and certain chemokines thought to counteract profibrotic mediators. Replacement of these downregulated mediators is also a therapeutic strategy. ${ }^{\mathrm{A}}$ Mediators validated as profibrotic in the lung by loss-of-function studies in mice (see text).

terns of chemokine and/or cytokine expression in patient BAL which appear to correlate with progression of the fibrotic process. The group of profibrotic mediators shown in Table 2 are elevated in the BAL and tissues of several of the disorders listed in Table 1 , though the majority of studies have been done with patients thought to have IPF (18-24). Because individual chemokines, cytokines, and leukotrienes act at multiple points and regulate each others' expression levels, determining the importance of any single mediator among these patients remains a challenge. Furthermore, key events in matrix remodeling take place at sites of cell-matrix and cell-cell interaction not necessarily reflected by soluble mediator levels. Perhaps for these reasons, serial measurements of chemokines and other soluble mediators (Table 2) have so far not proven valuable in predicting disease progression or treatment response. Recent results in experimental models, discussed further below, also call into question the degree to which inflammation per se actually drives fibrosis. Nonetheless, to date, efforts to control pulmonary fibrosis have mostly targeted proinflammatory mediators found in Table 2 .

Importance of the provisional matrix. The recognition of an alveolitis in patients at risk of interstitial fibrosis led to careful pathological studies examining the relationship between alveolitis and interstitial fibrosis in diffuse alveolar damage, UIP, and other chronic fibrotic processes $(25,26)$. These studies indicated that a major pathway of interstitial fibrosis is organization of a provisional matrix that appears in the alveolus as a consequence of alveolar wall injury, its ultimate appearance as a thickened alveolar wall being due to incorporation of the alveolar fibrotic process by re-epithelialization
(Figure 1, Figure 2, a and c). This is not to say that the interstitium itself is unaffected; indeed, expansion of mesenchymal elements (meaning interstitial connective tissue) occurs early and prominently in lung injury. But the lung appears particularly robust in its development and organization of fibrinous, provisional alveolar matrices. The structure of the lung suggests a reason for this propensity. The airway and alveolar compartment is virtually an open space surrounded by the entire blood volume and separated from it by microscopic epithelial and endothelial barriers. The lung defends this precarious situation not only by shunting blood flow away from areas of low oxygen level (e.g., areas poorly ventilated after edema or hemorrhage occurs), but also by expressing high levels of the key procoagulant, tissue factor, promoting coagulation along alveolar and airway surfaces (Figure 2a). Both alveolar macrophages (AMs) and epithelial cells constitutively express tissue factor, which is released in lipid vesicles into the surfactant-rich airway lining fluid (27-29). The appearance of proteinaceous exudates in alveoli as a result of barrier breakdown provides substrate to empower thrombin activation and fibrin formation. Further thrombin formation is then normally limited by local thrombin activation of activated protein C (APC) which then degrades a key procoagulant, Factor V. Thrombin activity is blocked by complexing with plasma-derived antithrombin III. Concurrently, low but significant levels of the plasminogen activator urokinase are continuously released along alveolar surfaces to facilitate timely resolution of extensive fibrin deposition and associated provisional matrix proteins (30). This is important because the insoluble matrix which accumulates in alveolar spaces contains both chemotactic and growth factors to support an influx of fibroblasts and fibroproliferation. This reportedly occurs within days of plasma leakage into alveoli in ARDS (Figure 1) (31-33). The importance of this resolution pathway is highlighted by the spontaneous appearance of fibrin and lung fibrosis in mice as a consequence of deficiency of combined urokinase and tissue plasminogen activators (34). Thus, normal coordination of the activation and function of the cascades of coagulation and fibrinolysis facilitates resolution of proteinaceous exudates and/or blood from alveolar spaces (Figure 1, a and b). Parallel with resorption of proteinaceous deposits is the removal of other matrix components such as the hyaluronans through macrophage scavenger receptors, such as CD44, which otherwise further provokes a remodeling response (35).

Role of epithelial lining cells. The focus on the alveolus in the 1980s also led to greater attention to the biology of alveolar and airway epithelial cells in lung injury and repair. The epithelial barrier is a potent regulator of inflammation in the lung. In part this is because of the extensive network of dendritic cells interdigitated with epithelial cells along the entire conducting airway surfaces (36). But epithelial cells themselves have been demonstrated to release 


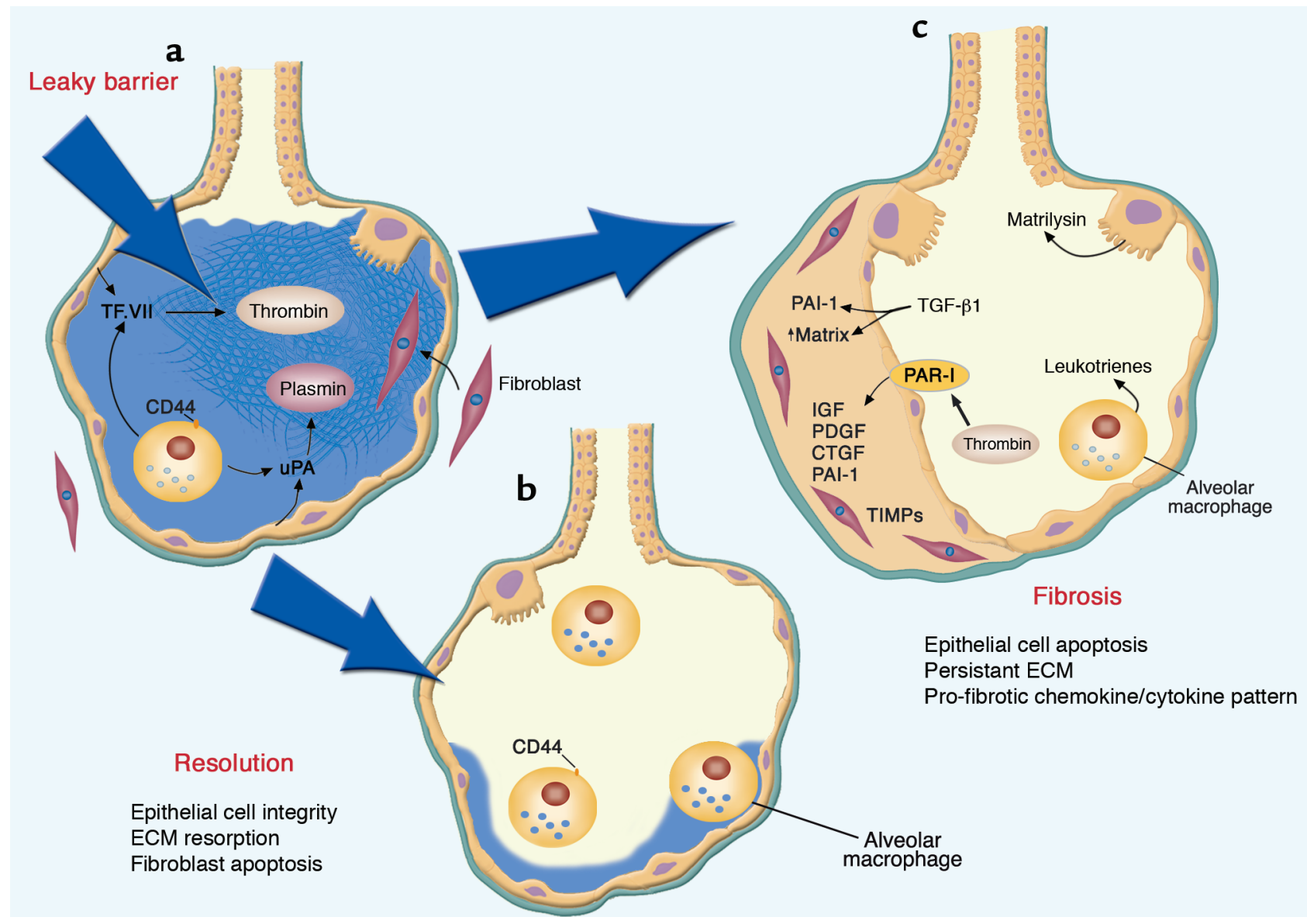

Figure 2

Determinants of lung response to breakdown in epithelial barrier function. (a) Coordinated processes of tissue factor/factor VII complexes initiating thrombin formation and plasminogen activation by urokinase (uPA). Plasmin facilitates initial exudate turnover. Ingrowth of fibroblasts and organization of exudates into a collagenous matrix occurs quickly. (b) Maintenance of epithelial integrity, resorption of ECMs by AMs, and fibroblast apoptosis favor resolution. (c) Epithelial cell apoptosis, activation of receptors of the profibrotic cytokines thrombin, leukotrienes, and TGF- $\beta_{1}$, and persistence of the ECM secondary to excess protease inhibitors (PAI-1 and tissue inhibitor of metalloprotease) with accumulation of fibroblast growth factors (IGF, PDGF, CTGF) favors fibrosis.

chemotactic factors and, along with macrophages, control the type of inflammatory cell influx into the lung. Also, alveolar epithelial cell death, mainly by apoptosis, is an early and consistent finding in these disorders, whether dramatic as in ARDS or very focal as in UIP or BO (37-39). Thus epithelial cells have emerged as a key site of initial injury as well as a major determinant of repair. The mechanisms of epithelial cell apoptosis in patients developing ARDS or UIP are still unclear but recent evidence points to the Fas/Fas ligand pathway (40-42). The implication that epithelial apoptosis is an early event is an important concept both because it can explain much of the breakdown of barrier function and because activation of the Fas signaling cascade culminating in apoptosis can also lead to release of pro-inflammatory mediators and overt inflammation (43). IL-8 expression and neutrophilic inflammation occur as a consequence of Fas activation in macrophages (44). Apoptosis, inflammation, and matrix remodeling in the lung appear intricately linked.
Finally, key signaling pathways through which the epithelium and mesenchyme (embryonic connective tissue) communicate to effect lung development have recently been found to reappear during lung injury (45). Though this field is still maturing, the prospects for new mechanistic insights are promising. Capitalizing on clues from invertebrate models, studies of vertebrate lung development have identified several mediators of epithelial and mesenchymal crosstalk, including sonic hedgehog $(\mathrm{SHH})$, epidermal growth factor (EGF), fibroblast growth factor 10 , TGF- $\beta_{1} /$ bone morphogenic protein, Wnt, fibronectin, and others (46-48). Each of these mediators has corresponding cellular receptors, which initiate signaling pathways. Integration of these signaling pathways orchestrates proliferation, migration, differentiation, and apoptosis of airway epithelial and mesenchymal cells during airway morphogenesis. At least two of these pathways, and likely others, are active during the response to lung injury in humans. $\mathrm{SHH}$, a secreted ligand of embryonic epithelial cells critical to early air- 


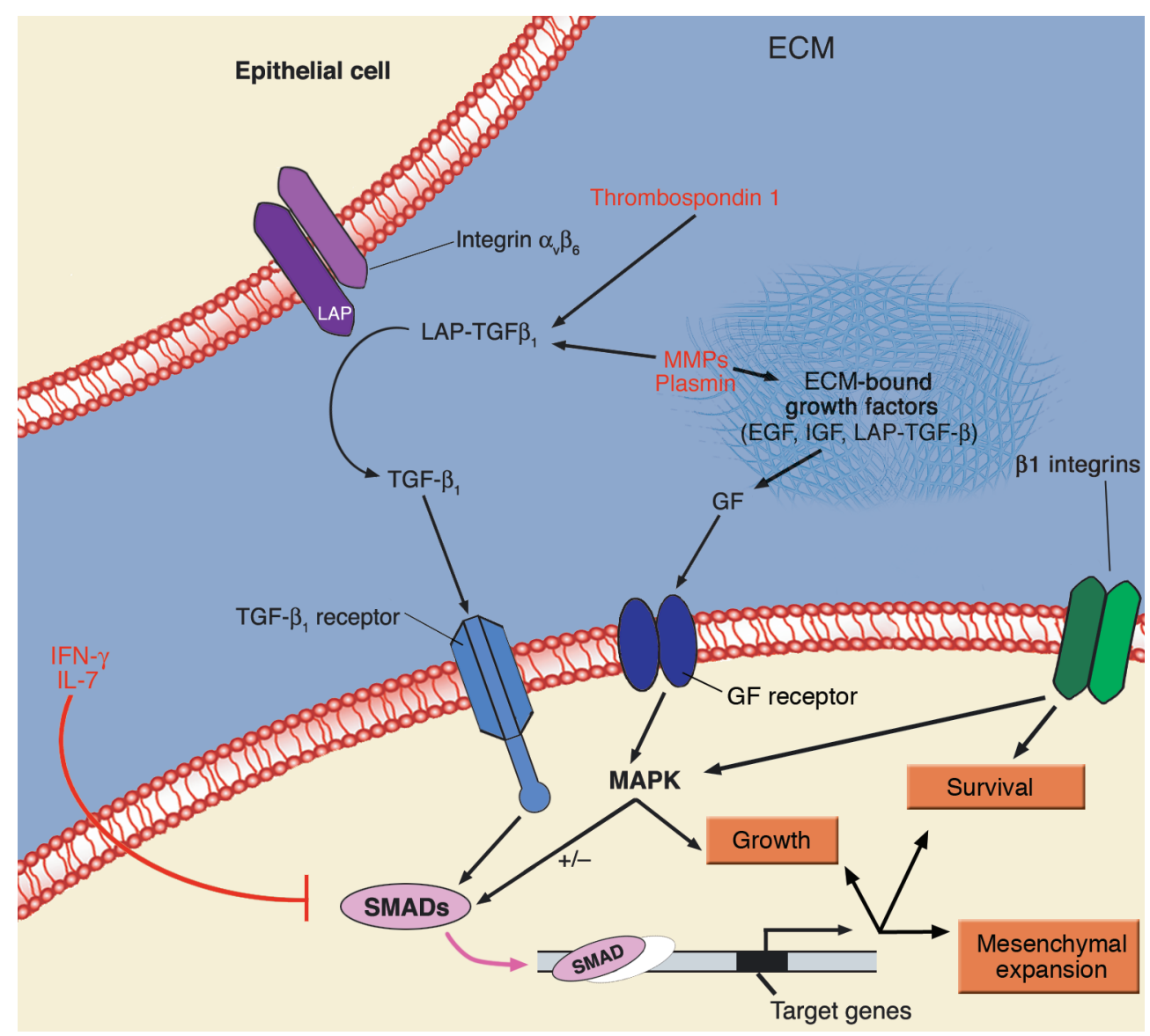

Figure 3

Pre- and postreceptor regulation of TGF- $\beta_{1}$ signaling. Latent TGF- $\beta_{1}$ can be activated by proteases (such asMMPs, plasmin) and by binding to TSP-1 or the integrin $\alpha_{v} \beta_{6}$, expressed on epithelial cells. Signaling through the TGF- $\beta_{1}$ receptor leads to phosphorylation of SMADs 2 and 3 and their translocation to the nucleus to mediate transcription of target genes. IFN- $\gamma$ and IL-7 induce counter-regulatory SMAD 7. Cellular responses to TGF- $\beta_{1}$ signaling are modulated by concurrent signaling through growth factor (GF) and adhesion receptors ( $\beta 1$ integrins). Integrated inputs from the pathways shown in the figure link deposition and turnover of ECM elements and GFs to expansion of mesenchymal elements such as fibroblasts and smooth muscle cells and the matrix proteins they secrete. Chemokines acting through $G$ protein-coupled receptors also modulate TF $\beta 1$ responses (not shown).

way formation, reappears in response to injury and promotes proliferation especially of neuroendocrine cells in the airway epithelium (49). SHH could also be expected to promote mesenchymal expansion, a point encouraging further investigation (50). Another pathway is signaling through $\beta$-catenin, a transcription factor connected to the cytoplasmic tails of epithelial adhesion proteins (cadherins) and implicated in promoting epithelial cell motility and matrix invasion (51). $\beta$-catenin was recently found by immunostaining in nuclei of hyperplastic epithelial cells and fibroblastlike cells within fibroblastic foci of UIP patients (52). Nuclear accumulation of $\beta$-catenin is a direct consequence of Wnt signaling, another critical signaling network for early lung development (53). A prominent downstream target of $\beta$-catenin signaling, MMP7 is highly expressed in lungs of UIP patients and has been shown to be an important protease in regulation of both epithelial cell apoptosis and epithelial cell migration $(54,55)$. Epithelial-mesenchymal transition (EMT), a consequence of $\beta$-catenin signaling, is a prominent pathway of matrix remodeling and fibrosis in injured kidney, liver, and breast tissues, where the appearance of matrix-secreting myofibroblasts derives in part from epithelial cells stimulated to undergo EMT through TGF- $\beta_{1}$, EGF, and Wnt $/ \beta$-catenin signaling pathways $(56,57)$. The evidence for an active Wnt pathway in lung matrix remodeling is rather preliminary, but an attractive concept in that EMT could promote epithelial-mesenchymal interactions not only by movement of fibroblastoid epithelial cells into contact with resident fibroblasts but also by promoting provisional matrix invasion and organization by epithelial cells undergoing this putative phenotypic switch. The documented involvement of signaling pathways known to be prominent in pulmonary fibrosis in the development of EMT in other tissues invites studies to explore the importance of this pathway in disorders of lung matrix remodeling.

In summary, early and repeated epithelial cell stimulation and injury, and its consequent interplay with inflammatory and mesenchymal cells, has emerged as 
a focal point in disorders of lung matrix remodeling (Figure 2). Therapeutic intervention, however, requires validation of the suspected molecular events. In this regard, targeted gene deficiency in mice has proven invaluable.

\section{Lessons learned from genetically altered mice}

Studies of pulmonary fibrosis in mice have largely used a model of drug-induced lung injury, bleomycininduced lung fibrosis. This model emanates from clinical observations that the use of bleomycin as an anticancer drug in humans is limited by development of widespread alveolar epithelial cell injury and development of pulmonary fibrosis (58). The mouse model has key elements of not only human bleomycin toxicity but also the prominent pathological features of the matrix remodeling disorders of unknown cause (Table 1): epithelial cell injury, inflammation and alveolar exudates, fibroblast activation and expansion with new collagen deposition, and thickened alveolar walls with reduced lung compliance. Also, like the human disorders, the intensity and progression of bleomycin injury is dependent on genetic background. Some mouse strains experience progressive fibrosis and in other models the process wanes and even reverses (59). What has been learned from studies of this model?

TGF- $\beta_{1}$. The cytokine most consistently linked both experimentally and by association in human and animal studies with tissue fibrosis is TGF- $\beta_{1}$. Indeed, microarray analysis of whole lung mRNA during bleomycin-induced fibrosis shows most of the known TGF- $\beta_{1}$-inducible genes to be upregulated (60). Overexpression of active TGF- $\beta_{1}$ along alveolar surfaces of mice leads to a vigorous fibrotic response, and inhibition of TGF- $\beta_{1}$ by antibodies or decoys abrogates bleomycin-induced fibrosis (61). TGF- $\beta_{1}$ 's activation, signaling, and actions to promote fibrosis are complicated but important to consider in the context of current and future clinical interventions.

TGF- $\beta_{1}$ is synthesized as an inactive precursor, but its subsequent activation is rather complex. Initial cleavage of pro-TGF- $\beta_{1}$ results in an inactive complex of mature TGF- $\beta_{1}$ and its propiece (termed the latencyassociated peptide, LAP). Full activation and competency for receptor engagement require an additional step to remove LAP. As indicated in Figure 3, there are at least three distinct mechanisms for TGF- $\beta_{1}$ activation, and studies in animals suggest that all of them are potentially active in the lung. In the bleomycin-induced lung fibrosis model the key pathway of TGF- $\beta$ activation appears to be through binding of LAP/ TGF- $\beta_{1}$ to the epithelial cell integrin $\alpha_{v} / \beta_{6}$ (illustrated in Figure 3 ) and its subsequent activation by a presumed conformational change mediated by the integrin and leading to presentation of active TGF- $\beta_{1}$ to TGF- $\beta_{1}$ receptors on adjacent cells (62). Importantly, LAP contains a typical integrin-binding sequence (arginine-glycine-aspartate). Munger and colleagues found that mice deficient in the beta 6 integrin, expressed exclusively in epithelial cells, were virtually completely protected from bleomycin-induced lung fibrosis, implying the integrin pathway is dominant in vivo. TGF- $\beta_{1}$ can also be activated by at least two additional pathways: binding of LAP/TGF $\beta$ to the matrix protein thrombospondin- 1 (TSP-1), again releasing TGF- $\beta_{1}$, and proteolytic cleavage of LAP by plasmin and certain metalloproteases $(63,64)$. Therefore regulation of integrin $\alpha_{v} / \beta_{6}$ expression and activation in alveolar epithelial cells of TSP-1 deposition and turnover in provisional matrices during injury, and the proteolytic activities of plasmin and metalloproteases, may all influence the levels of active TGF- $\beta_{1}$ in the lung. Yet another level of regulation is the spatial distribution of TGF- $\beta_{1}$, regulated by a set of proteins capable of binding both TGF- $\beta_{1}$ and the extracellular matrix (ECM): latent TGF-binding proteins (LTBPs) (65). The intricacy of regulation of TGF- $\beta_{1}$ activation attests to the critical importance the level of active TGF- $\beta_{1}$ has on lung biology.

As illustrated in Figure 3, the consequences of the binding of TGF- $\beta_{1}$ to its receptor are also complex. Once binding to the serine-threonine kinase receptor occurs, a signaling complex is formed, leading to the phosphorylation of SMADs 2 and 3 , the main cytoplasmic protein mediators of TGF- $\beta_{1}$ signaling, and their subsequent trafficking as a complex to the nucleus where they bind a well-defined SMAD-response element (66). Because direct DNA binding by SMADs is relatively weak, the SMADs must organize a nuclear transcriptional complex consisting of coactivators and the SMADs, which together initiate new gene transcription. All steps in this process appear to be elaborately regulated, and thus the consequences of SMAD signaling depend on the context in which it occurs. For example, TGF- $\beta_{1}$ signaling has different effects in embryonic and adult lung. Active TGF- $\beta_{1}$ suppresses alveolar epithelial cell proliferation. In the embryonic lung, TGF- $\beta_{1}$ overexpression leads to hypoplasia and lack of alveolar development (67). In the adult lung, TGF- $\beta_{1}$ overexpression leads to progressive fibrosis (68).

One key step of SMAD regulation is the expression of inhibitory SMAD elements, mainly SMADs 6 and 7, which act by preventing productive complex formation between SMADs 2 and 3 (Figure 3) (66). Potent inducers of SMAD7 are IFN- $\gamma$ and IL-7 (69). Induction of inhibitory SMADs by IFN- $\gamma$ is important in the interplay between TGF- $\beta_{1}$ and these cytokines in immune cell function. Although there are a number of rationales for using IFN- $\gamma$ in the treatment of patients with IPF, blockade of TGF- $\beta_{1}$ signaling may be the most compelling. Unfortunately, to date there is no information available as to whether lung TGF- $\beta_{1}$ signaling is altered in patients given IFN- $\gamma$ infusions $(70,71)$.

TGF- $\beta_{1}$ signaling is also critically regulated by integration with other cell signaling pathways (highlighted in Figure 3). One key point of intersection among signaling intermediates is MAP kinase activation occurring following engagement of growth factors, integrins, and even chemokine receptors (72-76). In most cases, acti- 
vated MAP kinases promote TGF- $\beta_{1}$ 's actions to enhance cell migration and mesenchymal expansion. Thus determinants of growth factor receptor activation, integrin signaling, and the pattern of cytokine/chemokine signaling all influence the response of cells to TGF- $\beta_{1}$. A future challenge is to determine critical points where therapeutic manipulation of active TGF- $\beta_{1}$ levels, or signaling pathways which influence the cells' response to TGF- $\beta_{1}$, will ameliorate excessive mesenchymal expansion without deleterious side effects.

Plasmin and thrombin. One gene highly induced in response to TGF- $\beta_{1}$ is the plasminogen activator inhibitor PAI-1. Mice deficient in PAI-1 are protected from, and mice overproducing PAI- 1 are more susceptible to, bleomycin-induced lung fibrosis $(77,78)$. Further, inducible expression of the plasminogen activator urokinase in the alveolar compartment ameliorates fibrosis in this model (79). These findings demonstrate that the urokinase/plasmin/PAI-1 system is functionally important in lung matrix remodeling. These animal studies are consistent with many pathological and biochemical observations in humans with ARDS, UIP, scleroderma, and sarcoidosis, all of which indicate an activation of both procoagulant and antifibrinolytic pathways (Figure 2) in these disorders $(28,80,81)$. Recent studies in mice, however, reveal that turnover of fibrin as the principal component of a provisional matrix does not explain the functional role of PAI- 1 and plasmin in matrix remodeling: fibrinogen null mice develop bleomycin-induced fibrosis comparably to wild-type mice, and yet PAI-1 still promotes the process (82). Thus the urokinase/PAI-1/plasmin system must also act at other sites, possibly by affecting mediator turnover or matrix-integrin signaling (Figure 3).

Thrombin is also a key player in the response of the lung to injury, with evidence of increased thrombin activation consistently seen in the BAL of fibrosis patients. Apart from its initial role in fibrinous matrix formation (Figure 2a), thrombin signaling through its cognate receptor, especially protease-activated receptor-1 (PAR-1), evokes production of secondary profibrotic cytokines such as IL-1 $\beta$ and connective tissue growth factor (CTGF) $(83,84)$. Inhibition of thrombin activity suppresses fibrosis in the bleomycininduced model of lung fibrosis in both rats and mice, supporting a mechanistic role for thrombin in tissue repair in the lung similar to that found at other sites of injury (85). Recently, enhanced expression of PAR1 in the epithelial cells of patients with UIP was reported (86). Enhanced thrombin formation persists in the lung of patients with disordered remodeling not only because of enhanced tissue factor activity but also because patients with UIP, sarcoidosis, and CVDs have lower levels of APC in their lavage fluid and enhanced levels of an APC inhibitor (87). Coordinated expression of the APC inhibitor and PAR-1 by epithelial cells likely acts to promote wound repair, but in the lung this also appears to favor excessive matrix expansion (Figure 2c).
Lipid mediators and chemokines. Studies in mice with null mutations in lipid mediator synthesis point to this family of mediators as important to matrix remodeling. Mice deficient in cyclo-oxygenase-2, required for prostaglandin E2 (PGE2) synthesis, showed enhanced fibrosis in response to bleomycin challenge (88). Conversely, mice deficient in phospholipase A2 (required for synthesis of all lipid mediators) or 5-lipoxygenase synthetase (required for leukotriene synthesis) show attenuated fibrotic responses to bleomycin $(89,90)$. These findings support the idea that PGE2 could suppress, and leukotrienes enhance, matrix remodeling leading to fibrosis. This conclusion in the murine model mirrors earlier conclusions from studies of isolated fibroblasts and tissues from patients with IPF (91). The mechanism(s) underlying this set of data, however, is (are) uncertain and likely complex. It is possible that these mediators ultimately act at least in part through regulation of the TGF- $\beta_{1}$ signaling pathway (92).

Consistently, studies of null mutations in cytokine and chemokine pathways in mice and the bleomycin model show a lack of correlation between regulation of the inflammatory response and matrix remodeling. Mice deficient in the CC chemokine receptor 2, the receptor for monocyte chemotactic protein-1 (MCP-1), show an attenuated fibrotic response to bleomycin but no change in any indices of acute or chronic inflammation $(21,93)$. Similarly, mice deficient in plateletactivating factor show defective acute inflammatory responses to bleomycin injection but no change in the overall degree of fibrosis. Inflammation in the lungs of integrin $\beta 6$-null mice was enhanced, even though fibrosis was blocked (62). These observations highlight again the distinctions between regulatory pathways of inflammation and the critical molecular events leading to matrix accumulation and progressive fibrosis. This conclusion is endorsed by the clinical experience that anti-inflammatory agents are relatively ineffective in blocking progressive fibrosis in most disorders of matrix remodeling. Validation in mice, as summarized above, by loss-of-function studies of several of the profibrotic mediators prominent in human disorders of matrix remodeling (indicated by note $\mathrm{A}$ in Table 2) offers new alternative approaches to this problem.

\section{Disorders of matrix remodeling: lessons from human mutations}

UIP. Although there is little risk to relatives for most patients with UIP, recent studies of small subgroups of patients with familial forms of pulmonary fibrosis are informative. Two families carrying separate mutations in the surfactant protein C (SPC) gene have been reported with progressive pneumonitis and lung fibrosis. SPC, a normal component of alveolar surfactant, is synthesized in a precursor form requiring C-terminal proteolytic processing for proper folding, assembly with lipid, and secretion. Nogee et al. reported childhood onset of interstitial pneumonitis and pulmonary fibrosis in an affected parent within a family carrying an SPC muta- 
tion (94). The mutation resulted in a truncated form of SPC that accumulated in the endoplasmic reticulum of type II alveolar cells. A survey of familial pulmonary fibrosis subjects identified at least one large kindred showing an autosomal dominant pattern of linkage to chromosome 6 in the region of the SPC gene (95). A single amino acid change, L188N, in the C-terminal SPC region was found in all six available family members with disease, but also in two unaffected obligate heterozygotes, and in none of four unaffected siblings or any of 88 unrelated control chromosomes. Expression of this mutant in murine alveolar cells showed lipid accumulation and toxicity, suggesting, as had the truncation mutant, that normal SPC function is critical to the health of type II cells. Studies of mice null for SPC also show pneumonitis and lung injury, confirming that loss of SPC function per se is sufficient to cause disease (96). Interestingly, the effects of SPC deficiency on lung injury were strongly influenced by the genetic background of the mice. This parallels the kindred with the $\mathrm{L} 188 \mathrm{~N}$ transversion, in which different subjects had markedly different histological patterns on lung biopsy, disease severity, and age of disease onset, implying that expression of the risk incurred by mutations in SPC is dependent on input from other genes.

Hermansky-Pudlak syndrome. This syndrome is a triad of albinism (of variable penetrance), platelet dysfunction with bleeding tendencies, and progressive lysosomal accumulation of ceroid lipids. The syndrome is due to an autosomal recessive mutation in any one of several proteins that were recently identified as forming a cytoplasmic protein complex involved in lysosome-organelle genesis. Thus the basic defect in these patients appears to be in lysosome formation and trafficking. A subset of these patients, especially those of Puerto Rican heritage, develop progressive pulmonary fibrosis, typically presenting in the mid-30s, and die of respiratory failure (97). The histological pattern, though not its distribution, is reminiscent of UIP. Recent studies identify lysosomal distortion and cellular toxicity of alveolar type II cells in Hermansky Pudlak patients (98). The type II cells show enlarged lysosomal granules engorged with surfactant. The studies point to injury of type II cells as an early and likely causal event in the initiation of the fibrotic process, reminiscent of the cellular changes seen in patients with mutations in the SPC gene.

Together these studies of patients with rare but defined mutations leading to progressive fibrosis confirm two main observations in the broad range of patients with disordered matrix remodeling: the importance of epithelial cells as a focal point of repeated injury and the strong influence of genetic background on disease progression. This underscores the importance of further detailed studies of genetic variation and disease progression, not only in UIP, but in all the disorders listed in Table 1. Reports of polymorphisms, mainly single nucleotide polymorphisms (SNPs), associated with many of these diseases are already begin- ning to appear in the literature, but much more experience and ultimately validation of hypotheses resulting from SNP analyses await.

\section{Conclusion}

From a clinical perspective, a crucial issue in managing patients with disorders of matrix remodeling is predicting which patients will develop more progressive disease. The current classification of interstitial pneumonias, reflecting radiographic patterns, histopathological evaluations, and pulmonary function testing, is focused on this issue. A radiographic and biopsy pattern of nonspecific interstitial pneumonia (NSIP), coupled with preserved ( $>50 \%$ predicted) and relatively stable vital capacity, conveys a much better prognosis than UIP even though it is quite uncertain whether NSIP is a disorder or only a pattern of persistent inflammation somewhere between resolution and progression (99, 100). In a similar vein, COP responds to corticosteroids, whereas UIP, diffuse alveolar damage, and BO do not. At the moment these important clinical distinctions have no molecular explanation. While all disorders of lung matrix remodeling appear to share common pathways of propagation, the different inciting events must be a determinant of progression. These are also not understood. And yet these issues are energized by the ongoing pace of elucidation of critical molecular pathways in epithelial injury, matrix remodeling, and signaling within the mesenchyme as reviewed here. These insights have already had medical impact. The focus of newer clinical trials, such as IFN- $\gamma$ for patients with IPF (70), has turned away from agents that nonspecifically block inflammation and toward mediators proven by experimental models to be involved in fibroproliferation and matrix remodeling. The promise of genomic approaches at both the experimental and clinical levels augurs for new therapeutic approaches based on these insights in the not distant future. In the meantime, better understanding of the process will likely lead to new screening tools to establish risk and predict disease progression (101-103).

\section{Acknowledgments}

The author thanks his many colleagues at the University of California at San Francisco for helpful discussions regarding this manuscript. This work is supported in part by NIH grant HL-44712.

\footnotetext{
1. Jeffery, P.K. 2001. Remodeling in asthma and chronic obstructive lung disease. Am. J. Respir. Crit. Care Med. 164:S28-S38.

2. Tiddens, H., Silverman, M., and Bush, A. 2000. The role of inflammation in airway disease: remodeling. Am. J. Respir. Crit. Care Med. 162:S7-S10. 3. Green, F.H. 2002. Overview of pulmonary fibrosis. Chest. 122:334S-339S 4. Heffner, J.E., Brown, L.K., Barbieri, C.A., Harpel, K.S., and DeLeo, J. 1995 Prospective validation of an acute respiratory distress syndrome predictive score. Am. J. Respir. Crit. Care Med. 152:1518-1526.

5. Martin, C., Papazian, L., Payan, M.J., Saux, P., and Gouin, F. 1995. Pulmonary fibrosis correlates with outcome in adult respiratory distress syndrome. A study in mechanically ventilated patients. Chest. 107:196-200.

6. Tomashefski, J.F., Jr. 2000. Pulmonary pathology of acute respiratory distress syndrome. Clin. Chest Med. 21:435-466.

7. Shimabukuro, D.W., Sawa, T., and Gropper, M.A. 2003. Injury and repair in lung and airways. Crit. Care Med. 31:S524-S531.
} 
8. 2002. American Thoracic Society and European Respiratory Society. American Thoracic Society/European Respiratory Society international multidisciplinary consensus classification of the idiopathic interstitial pneumonias. This joint statement of the American Thoracic Society (ATS), and the European Respiratory Society (ERS) was adopted by the ATS board of directors, June 2001, and by the ERS Executive Committee, June 2001. Am. J. Respir. Crit. Care Med. 165:277-304.

9. Sulica, R., Teirstein, A., and Padilla, M.L. 2001. Lung transplantation in interstitial lung disease. Curr. Opin. Pulm. Med. 7:314-322.

10. Estenne, M., and Hertz, M.I. 2002. Bronchiolitis obliterans after human lung transplantation. Am. J. Respir. Crit. Care Med. 166:440-444.

11. Pandit-Bhalla, M., Diethelm, L., Ovella, T., Sloop, G.D., and Valentine, V.G. 2003. Idiopathic interstitial pneumonias: an update. J. Thorac. Imaging. 18:1-13.

12. Weinberger, S.E., et al. 1978. Bronchoalveolar lavage in interstitial lung disease. Ann. Intern. Med. 89:459-466.

13. Keogh, B.A., and Crystal, R.G. 1982. Alveolitis: the key to the interstitial lung disorders. Thorax. 37:1-10.

14. Peters-Golden, M. 2003. Arachidonic acid metabolites: potential mediators and therapeutic targets in fibrotic lung disease. In Idiopathic pulmonary fibrosis. J.P. Lynch III, editor. Marcel Dekker Inc. New York, New York, USA. 421-452.

15. Phan, S.H., McGarry, B.M., Loeffler, K.M., and Kunkel, S.L. 1988. Binding of leukotriene $\mathrm{C} 4$ to rat lung fibroblasts and stimulation of collagen synthesis in vitro. Biochemistry. 27:2846-2853.

16. Yamamoto, T., Eckes, B., Mauch, C., Hartmann, K., and Krieg, T. 2000. Monocyte chemoattractant protein-1 enhances gene expression and synthesis of matrix metalloproteinase- 1 in human fibroblasts by an autocrine IL-1 alpha loop. J. Immunol. 164:6174-6179.

17. Pittet, J.F., et al. 2001. TGF-beta is a critical mediator of acute lung injury. J. Clin. Invest. 107:1537-1544.

18. Keane, M.P., et al. 2002. Imbalance in the expression of CXC chemokines correlates with bronchoalveolar lavage fluid angiogenic activity and procollagen levels in acute respiratory distress syndrome. J. Immunol. 169:6515-6521.

19. Kunkel, S.L., Lukacs, N., and Strieter, R.M. 1995. Chemokines and their role in human disease. Agents Actions Suppl. 46:11-22.

20. Smith, R.E., et al. 1995. A role for C-C chemokines in fibrotic lung disease. J. Leukoc. Biol. 57:782-787.

21. Belperio, J.A., et al. 2001. Critical role for the chemokine MCP-1/CCR2 in the pathogenesis of bronchiolitis obliterans syndrome. J. Clin. Invest 108:547-556. doi:10.1172/JCI200112214.

22. Krein, P.M., Sabatini, P.J., Tinmouth, W., Green, F.H., and Winston, B.W. 2003. Localization of insulin-like growth factor-I in lung tissues of patients with fibroproliferative acute respiratory distress syndrome. Am. J. Respir. Crit. Care Med. 167:83-90.

23. Reichenberger, F., et al. 2001. Different expression of endothelin in the bronchoalveolar lavage in patients with pulmonary diseases. Lung. 179:163-174

24. Homma, S., et al. 1995. Localization of platelet-derived growth factor and insulin-like growth factor I in the fibrotic lung. Am. J. Respir. Crit. Care Med. 152:2084-2089.

25. Kuhn, C., III, et al. 1989. An immunohistochemical study of architectural remodeling and connective tissue synthesis in pulmonary fibrosis. Am. Rev. Respir. Dis. 140:1693-1703.

26. Fukuda, Y., et al. 1987. The role of intraalveolar fibrosis in the process of pulmonary structural remodeling in patients with diffuse alveolar damage. Am. J. Pathol. 126:171-182.

27. Gross, T.J., Simon, R.H., and Sitrin, R.G. 1992. Tissue factor procoagulant expression by rat alveolar epithelial cells. Am. J. Respir. Cell Mol. Biol. 6:397-403.

28. Idell, S. 2003. Coagulation, fibrinolysis, and fibrin deposition in acute lung injury. Crit. Care Med. 31:S213-S220.

29. Chapman, H.A., Stahl, M., Allen, C.L., Yee, R., and Fair, D.S. 1988. Regulation of the procoagulant activity within the bronchoalveolar compartment of normal human lung. Am. Rev. Respir. Dis. 137:1417-1425.

30. Marshall, B.C., et al. 1991. Alveolar epithelial cells express both plasminogen activator and tissue factor. Potential role in repair of lung injury. Chest. 99:25S-27S

31. Marshall, R.P., et al. 2000. Fibroproliferation occurs early in the acute respiratory distress syndrome and impacts on outcome. Am. J. Respir. Crit. Care Med. 162:1783-1788.

32. Pugin, J., Verghese, G., Widmer, M.C., and Matthay, M.A. 1999. The alveolar space is the site of intense inflammatory and profibrotic reactions in the early phase of acute respiratory distress syndrome. Crit. Care Med. 27:304-312.

33. Chesnutt, A.N., Matthay, M.A., Tibayan, F.A., and Clark, J.G. 1997. Early detection of type III procollagen peptide in acute lung injury. Pathogenetic and prognostic significance. Am. J. Respir. Crit. Care Med. 156:840-845.

34. Carmeliet, P., et al. 1994. Physiological consequences of loss of plasminogen activator gene function in mice. Nature. 368:419-424.

35. Teder, P., et al. 2002. Resolution of lung inflammation by CD44. Science. 296: $155-158$.
36. Lambrecht, B.N., Prins, J.B., and Hoogsteden, H.C. 2001. Lung dendritic cells and host immunity to infection. Eur. Respir. J. 18:692-704.

37. Martin, T.R., Nakamura, M., and Matute-Bello, G. 2003. The role of apoptosis in acute lung injury. Crit. Care Med. 31:S184-S188.

38. Uhal, B.D. 2002. Apoptosis in lung fibrosis and repair. Chest. 122:293S-298S

39. Kuwano, K., et al. 1999. The involvement of Fas-Fas ligand pathway in fibrosing lung diseases. Am. J. Respir. Cell Mol. Biol. 20:53-60.

40. Albertine, K.H., et al. 2002. Fas and fas ligand are up-regulated in pulmonary edema fluid and lung tissue of patients with acute lung injury and the acute respiratory distress syndrome. Am. J. Pathol. 161:1783-1796.

41. Kuwano, K., et al. 2002. Increased circulating levels of soluble Fas ligand are correlated with disease activity in patients with fibrosing lung diseases. Respirology. 7:15-21.

42. Wang, R., Zagariya, A., Ang, E., Ibarra-Sunga, O., and Uhal, B.D. 1999 Fas-induced apoptosis of alveolar epithelial cells requires ANG II generation and receptor interaction. Am. J. Physiol. 277:L1245-L1250.

43. Chen, J.J., Sun, Y., and Nabel, G.J. 1998. Regulation of the proinflam matory effects of Fas ligand (CD95L). Science. 282:1714-1717.

44. Park, D.R., et al. 2003. Fas (CD95) induces proinflammatory cytokine responses by human monocytes and monocyte-derived macrophages. J. Immunol. 170:6209-6216.

45. Warburton, D., et al. 2001. Do lung remodeling, repair, and regeneration recapitulate respiratory ontogeny? Am. J. Respir. Crit. Care Med. 164:S59-S62.

46. Warburton, D., Zhao, J., Berberich, M.A., and Bernfield, M. 1999. Molecular embryology of the lung: then, now, and in the future. Am. J. Physiol. 276:L697-L704.

47. Costa, R.H., Kalinichenko, V.V., and Lim, L. 2001. Transcription factors in mouse lung development and function. Am. J. Physiol. Lung Cell. Mol. Physiol. 280:L823-L838.

48. Sakai, T., Larsen, M., and Yamada, K.M. 2003. Fibronectin requirement in branching morphogenesis. Nature. 423:876-881.

49. Watkins, D.N., et al. 2003. Hedgehog signalling within airway epithelial progenitors and in small-cell lung cancer. Nature. 422:313-317.

50. Pepicelli, C.V., Lewis, P.M., and McMahon, A.P. 1998. Sonic hedgehog regulates branching morphogenesis in the mammalian lung. Curr. Biol. 8:1083-1086

51. Muller, T., Bain, G., Wang, X., and Papkoff, J. 2002. Regulation of epithelial cell migration and tumor formation by beta-catenin signaling. Exp. Cell Res. 280:119-133.

52. Chilosi, M., et al. 2003. Aberrant Wnt/beta-catenin pathway activation in idiopathic pulmonary fibrosis. Am. J. Pathol. 162:1495-1502.

53. Morrisey, E.E. 2003. Wnt signaling and pulmonary fibrosis. Am. J. Pathol. 162:1393-1397.

54. Dunsmore, S.E., et al. 1998. Matrilysin expression and function in airway epithelium. J. Clin. Invest. 102:1321-1331.

55. Zuo, F., et al. 2002. Gene expression analysis reveals matrilysin as a key regulator of pulmonary fibrosis in mice and humans. Proc. Natl. Acad. Sci. U. S. A. 99:6292-6297.

56. Iwano, M., et al. 2002. Evidence that fibroblasts derive from epithelium during tissue fibrosis. J. Clin. Invest. 110:341-350. doi:10.1172/JCI200215518.

57. Yang, J., and Liu, Y. 2001. Dissection of key events in tubular epithelial to myofibroblast transition and its implications in renal interstitial fibrosis. Am. J. Pathol. 159:1465-1475.

58. Adamson, I.Y. 1984. Drug-induced pulmonary fibrosis. Environ. Health Perspect. 55:25-36.

59. Haston, C.K., et al. 2002. Bleomycin hydrolase and a genetic locus within the MHC affect risk for pulmonary fibrosis in mice. Hum. Mol. Genet. 11:1855-1863.

60. Kaminski, N., et al. 2000. Global analysis of gene expression in pulmonary fibrosis reveals distinct programs regulating lung inflammation and fibrosis. Proc. Natl. Acad. Sci. U. S. A. 97:1778-1783.

61. Kelly, M., Kolb, M., Bonniaud, P., and Gauldie, J. 2003. Re-evaluation of fibrogenic cytokines in lung fibrosis. Curr. Pharm. Des. 9:39-49.

62. Munger, J.S., et al. 1999. The integrin alpha v beta 6 binds and activates latent TGF beta 1: a mechanism for regulating pulmonary inflammation and fibrosis. Cell. 96:319-328.

63. Murphy-Ullrich,J.E., and Poczatek, M. 2000. Activation of latent TGF-beta by thrombospondin-1: mechanisms and physiology. Cytokine Growth Factor Rev. 11:59-69.

64. Mu, D., et al. 2002. The integrin alpha(v)beta8 mediates epithelial homeostasis through MT1-MMP-dependent activation of TGF-beta1. J. Cell Biol. 157:493-507.

65. Dallas, S.L., Rosser, J.L., Mundy, G.R., and Bonewald, L.F. 2002. Proteolysis of latent transforming growth factor-beta (TGF-beta)-binding protein-1 by osteoclasts. A cellular mechanism for release of TGF-beta from bone matrix. J. Biol. Chem. 277:21352-21360.

66. Massague, J., and Chen, Y.G. 2000. Controlling TGF-beta signaling. Genes Dev. 14:627-644.

67. Zeng, X., Gray, M., Stahlman, M.T., and Whitsett, J.A. 2001. TGF-beta1 
perturbs vascular development and inhibits epithelial differentiation in fetal lung in vivo. Dev. Dyn. 221:289-301.

68. Gauldie, J., Sime, P.J., Xing, Z., Marr, B., and Tremblay, G.M. 1999. Transforming growth factor-beta gene transfer to the lung induces myofibroblast presence and pulmonary fibrosis. Curr. Top. Pathol. 93:35-45.

69. Huang, M., et al. 2002. IL-7 inhibits fibroblast TGF- $\beta$ production and signaling in pulmonary fibrosis. J. Clin. Invest. 109:931-937. doi:10.1172/ JCI200214685.

70. Ziesche, R., Hofbauer, E., Wittmann, K., Petkov, V., and Block, L.H. 1999 A preliminary study of long-term treatment with interferon gamma-1b and low-dose prednisolone in patients with idiopathic pulmonary fibrosis. N. Engl. J. Med. 341:1264-1269.

71. Kalra, S., Utz, J.P., and Ryu, J.H. 2003. Interferon gamma-1b therapy for advanced idiopathic pulmonary fibrosis. Mayo Clin. Proc. 78:1082-1087.

72. Thannickal, V.J., et al. 2003. Myofibroblast differentiation by transforming growth factor-beta 1 is dependent on cell adhesion and integrin signaling via focal adhesion kinase. J. Biol. Chem. 278:12384-12389.

73. Bhowmick, N.A., Zent, R., Ghiassi, M., McDonnell, M., and Moses, H.L. 2001. Integrin beta 1 signaling is necessary for transforming growth factor-beta activation of p38MAPK and epithelial plasticity. J. Biol. Chem. 276:46707-46713

74. Hayashida, T., Decaestecker, M., and Schnaper, H.W. 2003. Cross-talk between ERK MAP kinase and Smad signaling pathways enhances TGF-beta-dependent responses in human mesangial cells. FASEB J. 17:1576-1578.

75. Chen, Y., et al. 2002. CTGF expression in mesangial cells: involvement of SMADs, MAP kinase, and PKC. Kidney Int. 62:1149-1159.

76. Janda, E., et al. 2002. Ras and TGF[beta] cooperatively regulate epithelial cell plasticity and metastasis: dissection of Ras signaling pathways. J. Cell Biol. 156:299-313.

77. Eitzman, D.T., et al. 1996. Bleomycin-induced pulmonary fibrosis in transgenic mice that either lack or overexpress the murine plasminogen activator inhibitor-1 gene. J. Clin. Invest. 97:232-237.

78. Olman, M.A., Mackman, N., Gladson, C.L., Moser, K.M., and Loskutoff, D.J. 1995. Changes in procoagulant and fibrinolytic gene expression during bleomycin-induced lung injury in the mouse. J. Clin. Invest. 96:1621-1630.

79. Sisson, T.H., et al. 2002. Inducible lung-specific urokinase expression reduces fibrosis and mortality after lung injury in mice. Am. J. Physiol. Lung Cell. Mol. Physiol. 283:L1023-L1032.

80. Kotani, I., et al. 1995. Increased procoagulant and antifibrinolytic activities in the lungs with idiopathic pulmonary fibrosis. Thromb. Res. 77:493-504.

81. Chapman, H.A., Allen, C.L., and Stone, O.L. 1986. Abnormalities in pathways of alveolar fibrin turnover among patients with interstitial lung disease. Am Rev. Respir. Dis. 133:437-443.

82. Hattori, N., et al. 2000. Bleomycin-induced pulmonary fibrosis in fibrinogen-null mice. J. Clin. Invest. 106:1341-1350.

83. Chambers, R.C., Leoni, P., Blanc-Brude, O.P., Wembridge, D.E., and Laurent, G.J. 2000. Thrombin is a potent inducer of connective tissue growth factor production via proteolytic activation of protease-activated receptor-1. J. Biol. Chem. 275:35584-35591.

84. Ruf, W., and Riewald, M. 2003. Tissue factor-dependent coagulation protease signaling in acute lung injury. Crit. Care Med. 31:S231-S237.
85. Howell, D.C., et al. 2001. Direct thrombin inhibition reduces lung collagen, accumulation, and connective tissue growth factor mRNA levels in bleomycin-induced pulmonary fibrosis. Am. J. Pathol. 159:1383-1395.

86. Howell, D.C., Laurent, G.J., and Chambers, R.C. 2002. Role of thrombin and its major cellular receptor, protease-activated receptor-1, in pulmonary fibrosis. Biochem. Soc. Trans. 30:211-216.

87. Kobayashi, H., et al. 1998. Protein C anticoagulant system in patients with interstitial lung disease. Am. J. Respir. Crit. Care Med. 157:1850-1854.

88. Keerthisingam, C.B., et al. 2001. Cyclooxygenase-2 deficiency results in a loss of the anti-proliferative response to transforming growth factorbeta in human fibrotic lung fibroblasts and promotes bleomycininduced pulmonary fibrosis in mice. Am. J. Pathol. 158:1411-1422.

89. Nagase, T., et al. 2002. A pivotal role of cytosolic phospholipase A(2) in bleomycin-induced pulmonary fibrosis. Nat. Med. 8:480-484.

90. Peters-Golden, M., et al. 2002. Protection from pulmonary fibrosis in leukotriene-deficient mice. Am. J. Respir. Crit. Care Med. 165:229-235.

91. Wilborn, J., et al. 1996. Constitutive activation of 5-lipoxygenase in the lungs of patients with idiopathic pulmonary fibrosis. J. Clin. Invest. 97:1827-1836.

92. Ricupero, D.A., Rishikof, D.C., Kuang, P.P., Poliks, C.F., and Goldstein R.H. 1999. Regulation of connective tissue growth factor expression by prostaglandin E(2). Am. J. Physiol. 277:L1165-L1171.

93. Moore, B.B., et al. 2001. Protection from pulmonary fibrosis in the absence of CCR2 signaling. J. Immunol. 167:4368-4377.

94. Nogee, L.M., et al. 2001. A mutation in the surfactant protein C gene associated with familial interstitial lung disease. N. Engl. J. Med. 344:573-579.

95. Thomas, A.Q., et al. 2002. Heterozygosity for a surfactant protein C gene mutation associated with usual interstitial pneumonitis and cellular nonspecific interstitial pneumonitis in one kindred. Am. J. Respir. Crit. Care Med. 165:1322-1328.

96. Glasser, S.W., et al. 2003. Pneumonitis and emphysema in sp-C gene targeted mice. J. Biol. Chem. 278:14291-14298.

97. Brantly, M., et al. 2000. Pulmonary function and high-resolution CT findings in patients with an inherited form of pulmonary fibrosis, Hermansky-Pudlak syndrome, due to mutations in HPS-1. Chest. 117:129-136.

98. Nakatani, Y., et al. 2000. Interstitial pneumonia in Hermansky-Pudlak syndrome: significance of florid foamy swelling/degeneration (giant lamellar body degeneration) of type-2 pneumocytes. Virchows Arch. 437:304-313.

99. Latsi, P.I., et al. 2003. Fibrotic idiopathic interstitial pneumonia: the prognostic value of longitudinal functional trends. Am. J. Respir. Crit. Care Med. 168:531-537.

100. Flaherty, K.R., et al. 2003. Fibroblastic foci in usual interstitial pneumonia: idiopathic versus collagen vascular disease. Am. J. Respir. Crit. Care Med. 167:1410-1415.

101. Ware, L.B., Fang, X., and Matthay, M.A. 2003. Protein C and thrombomodulin in human acute lung injury. Am. J. Physiol. Lung Cell. Mol. Physiol. 285:L514-L521.

102. Greene, K.E., et al. 2002. Serum surfactant proteins-A and -D as biomarkers in idiopathic pulmonary fibrosis. Eur. Respir. J. 19:439-446.

103. Takahashi, H., et al. 2000. Serum surfactant proteins A and D as prognostic factors in idiopathic pulmonary fibrosis and their relationship to disease extent. Am. J. Respir. Crit. Care Med. 162:1109-1114. 